\title{
Complete Superior Sagittal Sinus Thrombosis Along With Subarachnoid Hemorrhage During Early Postpartum Period, Presented by Left Hemiparesis: A Rare Case Report
}

\author{
Yaseer Muhammad Tareq Khan ${ }^{\mathrm{a}}$, Houda Nasser Al Yaqoubi ${ }^{\mathrm{b}}$, \\ Nishat Fatema ${ }^{\text {c, d }}$
}

\begin{abstract}
Cerebral venous sinus thrombosis (CVST) is a fatal neurological emergency during pregnancy and puerperium. We report a 25-yearold woman with superior sagittal sinus thrombosis (SSST) along with subarachnoid hemorrhage developed 4 days after an uncomplicated vaginal birth, presenting with a 3-day history of global headache and sudden onset of left-sided hemiparesis. After confirmatory diagnosis by magnetic resonance imaging (MRI) of the brain, she was treated with low molecular weight heparin (LMWH) followed by warfarin. She recovered within a few weeks and was regularly followed up. SSST may present during puerperium to the obstetrician and physician with various non-specific presentations, so physicians caring for women in the peripartum period should be familiar with the differential diagnosis and have a high index of suspicion for CVST with or without intracranial hemorrhage. A multidisciplinary approach is important to the management of this rare complication of pregnancy and puerperium.
\end{abstract}

Keywords: Sagittal sinus thrombosis; Subarachnoid hemorrhage; Postpartum period

\section{Introduction}

Cerebral venous sinus thrombosis (CVST) is a rare condition accounting for $0.5-1.0 \%$ of all strokes [1-3]. CVST is an uncommon complication in pregnancy and puerperium, with an incidence of $1: 10,000$ to $1: 25,000$ deliveries $[4,5]$. Mortality rates associated with CVST range from $6 \%$ to $10 \%$, and ap-

Manuscript submitted October 1, 2017, accepted October 13, 2017

${ }^{a}$ Department of Medicine, Ibri Regional Hospital, Ministry of Health, Oman bepartment of Obstetrics and Gynaecology, Ibri Regional Hospital, Ministry of Health, Oman

'Department of Obstetrics and Gynaecology, Ibri Regional Hospital, Ministry of Health, Oman

${ }^{\mathrm{d} C}$ Corresponding Author: Nishat Fatema, Department of Obstetrics and Gynaecology, Ibri Regional Hospital, Ministry of Health, Oman.

Email: nishat.doc.om@gmail.com

doi: https://doi.org/10.14740/jnr452w proximately $80-90 \%$ completely recover if appropriate and timely treatment is undertaken [6]. Pregnancy and puerperium are the known risk factors for CVST. Women are particularly vulnerable prior to delivery and during the postpartum period because of the hypercoagulable state that accompanies pregnancy. Apart from pregnancy and puerperium, the etiological factors for CVST include oral contraceptives, carcinoma, hypercoagulable state, leiomyoma, vasculitis, inflammatory bowel disease, head trauma, intracranial or systemic infections, dehydration, substance abuse, and Behcet's disease [5, 7].

Superior sagittal sinus thrombosis (SSST) including subarachnoid hemorrhage (SAH) is a rare complication during pregnancy and puerperium [8]. To the best of our knowledge of previous studies, of the CVST cases that developed within the perinatal period, only two cases of SSST presented with SAH: one in the post-cesarean state and the other after vaginal delivery. Only one case of SSST with SAH during pregnancy is reported in the literature [8-10]. Headache is the most frequent presenting symptom $(80-90 \%)$ of CVST, followed by seizures $(47 \%)$ and paresis $(43 \%)$. SSST may present with unilateral paralysis that extends to the other side, secondary to extension of the clot into the cerebral veins. Because of the location, this may present as a unilateral lower extremity weakness or paraplegia $[5,7]$. Various imaging techniques are used to confirm the diagnosis, with CT angiography and magnetic resonance imaging (MRI) being most sensitive and specific [6]. Anticoagulants are primarily used in the treatment of CVST developed during puerperal period $[11,12]$.

We report a case of a 25-year-old woman Para2Ab1, with SSST with SAH developed 4 days after an uncomplicated vaginal child birth. She present with a 3-day history of severe headache and sudden onset of left-sided hemiparesis. MRI demonstrated the presence of complete SSST with evidence of SAH in the left frontal sulci. She was treated with low molecular weight heparin (LMWH) followed by warfarin. She recovered a few weeks after admission and was regularly followed up.

\section{Case Report}

On the fourth day of her puerperal period, a 25 -year-old patient 
Table 1. Laboratory Test Results

\begin{tabular}{|c|c|}
\hline Investigations & Results \\
\hline \multicolumn{2}{|l|}{ Complete blood count (CBC) } \\
\hline Hemoglobin & $12.5 \mathrm{~g} / \mathrm{dL}$ \\
\hline Platelets & $306,000 / \mu \mathrm{L}$ \\
\hline Hemotocrit & $40.33 \%$ \\
\hline \multicolumn{2}{|l|}{ Renal function tests (RFTs) } \\
\hline Urea & $4 \mathrm{mmol} / \mathrm{L}(2.5-6.7)$ \\
\hline Creatinine & $49 \mu \mathrm{mol} / \mathrm{L}(45-90)$ \\
\hline \multicolumn{2}{|l|}{ Liver function tests (LFTs) } \\
\hline Albumin & $29 \mathrm{~g} / \mathrm{L}(35-50)$ \\
\hline \multicolumn{2}{|l|}{ Electrolyte } \\
\hline Sodium & 140 mmol/L (137 - 148) \\
\hline Potassium & $4.34 \mathrm{mmol} / \mathrm{L}(3.5-5)$ \\
\hline Random blood sugar (RBC) & $3.97 \mathrm{mmol} / \mathrm{L}(3-7.7)$ \\
\hline Anti nuclear antibody (ANA) & Non reactive \\
\hline Erythrocyte sedimentation rate (ESR) & $45 \mathrm{~mm} / \mathrm{h}$ \\
\hline \multicolumn{2}{|l|}{ Thyroid function tests (TFTs) } \\
\hline Thyroxin (T4) & $15.3 \mathrm{pmol} / \mathrm{L}(8.4-22.6)$ \\
\hline \multicolumn{2}{|l|}{ Anti phospholipid antibody screening } \\
\hline Anti-cardiolopin IgG & $8.0 \mathrm{U} / \mathrm{mL}(20 \mathrm{U} / \mathrm{mL})$ \\
\hline B2 glycoprotein $1 \mathrm{IgG}$ & $6.4(20 \mathrm{U} / \mathrm{mL})$ \\
\hline
\end{tabular}

presented to our accident and emergency department (A\&E) with complaints of left-sided hemiparesis following a 3-day history of global headache, dizziness, and vomiting. Patient Para2Ab1 had a body mass index of $32 \mathrm{~kg} / \mathrm{m}^{2}$ and had an uncomplicated vaginal delivery 4 days prior. Her antenatal period was uneventful. She was neither hypertensive nor diabetic. She had no signs of infection, deep vein thrombosis (DVT), or eclampsia during pregnancy. There was no significant past medical or surgical history.

On admission, she was found to be alert and oriented with normal mental status (Glasgow coma scale/score (GCS) level 15/15). Her temperature, respirations, blood pressure, and pulse rate were normal. She had left-sided hemiparesis with motor power grade $4 / 5$. There was no cranial nerve deficit, no facial asymmetry, no nystagmus or slurring of speech observed. During the ophthalmologic examination, her pupils were $2 \mathrm{~mm}$ in size, equal reacting to light and accommodation, and bilateral papilledema was observed. No abnormality was detected on other systemic examinations. Electrocardiography (ECG), echocardiogram (left ventricular ejection fraction (LVEF) 70\%) was revealed to be normal.

Her gynecologic examination was compatible with the postpartum period, and no abnormality was observed. All hematologic and cardiologic workups, including electrolytes, blood count, coagulation test, and renal function test were within the normal limits (Table 1). No characteristic was observed in the thrombophilia screening and rheumatoid factors of the patient (Table 1). 



Figure 1. (a) Brain CT scan showing evidence of subarachnoid hemorrhage in the frontal cortical sulci. (b) CT of the brain with arrow showing "typical empty delta sign". (c) CT brain in sagittal plane showing the lack of normal blood flow void outlined by arrows in the superior sagittal sinus.

An urgent contrast brain CT revealed complete SSST with an empty delta sign and left frontal SAH (Fig. $1 \mathrm{a}, \mathrm{b}$, c). To confirm the diagnosis, brain MRI was performed and showed dilatation of the superior sagittal sinus, superficial cortical veins that showed heterogenous high signal in T1, and multiple filling defects in magnetic resonance venography (MRV) images (Fig. 2 a, b, c). These findings were in favor of venous thrombosis of the superior sagittal sinus . $\mathrm{SAH}$ was also seen in the left frontal sulci. Brain parenchyma showed normal signals, with no evidence of ischemia or venous infarction.

We admitted the patient to the intensive care unit (ICU) and commenced her on anticoagulation with LMWH $(6,000$ IU bis in die (BID)). The patient's symptoms improved within a few days and thereafter she had an uneventful course. Heparin was changed to warfarin (4 mg, once daily (OD)) when the target international normalized ratio (INR) of 2.0 - 3 was achieved and she was followed up regularly.

\section{Discussion}

Although pregnancy and puerperium increase the risk of thromboembolic diseases, SSST is a rare complication of pregnancy or puerperium. It commonly occurs during the third trimester and puerperal period [13]. Headache $(80-90 \%)$ is the most common presenting symptom that could be isolated in up to $5 \%$ of cases. In the international study on cerebral vein and dural sinus thrombosis, focal or generalized seizures and paresis were seen in up to $40 \%$ of patients, changes of the mental status and aphasia were reported in around $20 \%$, and approximately $10 \%$ presented with coma [5].

Our case presented with a 3-day history of diffuse severe headache, dizziness, and subsequently developed left-sided
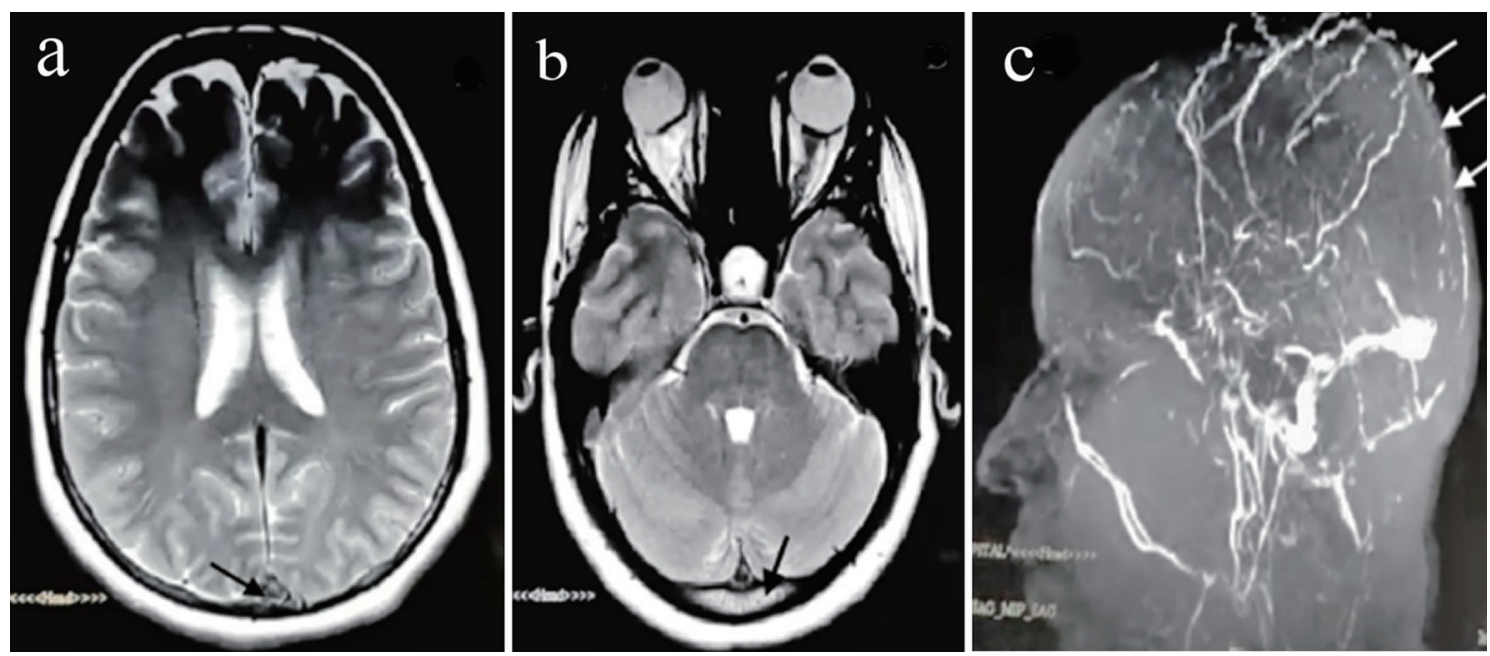

Figure 2. (a) T1-weighted MRI with axial plane showing a hyperintense signal from the thrombosed superior sagittal sinus. (b) $\mathrm{MRI}$ of the head in the coronal plane displaying a hyperintense signal from the thrombosed superior sagittal sinus. (c) Brain magnetic resonance angiography with venography in the sagittal plane showing interruption in blood flow in the superior sagittal sinus (arrow) caused by thrombus. 
hemiparesis on the fourth day of the postpartum period. A case reported by Chuidian et al included a patient at the 10th day of the postpartum period who initially presented with isolated headache, which was misdiagnosed as a non-specific headache and discharged home after symptomatic management. Six days later, the patient presented again with a worsening of the headache associated with seizures; a diagnosis of SSST was then made after a brain MRI [6]. As our patient presented with the complex symptoms rather than an isolated headache, we were able to make an early diagnosis of SSST and SAH with the aid of imaging techniques.

In recent years early diagnosis of CVST has been possible because of improved non-invasive imaging techniques [5]. CT angiography and MRI in combination with MRV is the choice of imaging modality for the diagnosis of CVST [6]. In relation to our case, a CT scan revealed complete thrombosis of the SSS with associated SAH within the frontal lobe. The typical empty delta sign was observed in contrast CT. In contrast axial CT images this sign is seen in only $20 \%$ of cases, as mentioned by Femi et al [5]. MRI and MRV of the brain revealed confirmation of SSST with SAH within the left frontal sulci.

Around $30 \%$ of patients may also have some form of hemorrhage with CVST identified on imaging; these are usually small, low-pressure intraparenchymal hemorrhages of venous origin [6]. In our case, SAH was detected along with SSST in brain CTs and MRIs. The presence of intracranial hemorrhage along with CVST is associated with increased hemorrhage risk.

Although there is an increase of intracranial bleeding risk and conflict of indications, heparin is the first option as an anticoagulant treatment despite the presence of intracranial bleeding for this group of patients [11]. In a study of 43 patients with CVST and intracranial hemorrhage, Einhaupl et al reported a mortality of $15 \%$ in heparin-treated patients in comparison to $69 \%$ in those who were not treated with heparin [14].

However, we also initially commenced anticoagulation therapy with LMWH for our patient and changed to warfarin when the target INR of 2.0 - 3 was achieved. The initial anticoagulation therapy for CVST is to prevent thrombus growth, to facilitate recanalization, and to prevent deep venous thrombosis or pulmonary embolism. Post-acute treatment with oral anticoagulants is recommended for 6 and up to 12 months [7].

Cerebral venous thrombosis associated with pregnancy and puerperium has a better prognosis than thrombosis due to other causes if immediate diagnosis and appropriate management is given before fatal consequences. The recurrence rate of CVST is about $2-7 \%$. The risk of recurrence of cerebral venous thrombosis in a subsequent pregnancy should be fully explained to patients and the prophylactic use of anticoagulants in preventing recurrence of CVST in future pregnancies should be discussed [13].

SSST may present during puerperium to the obstetrician and physician with various non-specific presentations. Physicians caring for women in the peripartum period should be familiar with the differential diagnosis and have a high index of suspicion for considering CVST, with or without SAH. A multidisciplinary approach is important to the management of this rare complication of pregnancy and puerperium [15].

\section{References}

1. Kashkoush AI, Ma H, Agarwal N, Panczykowski D, Tonetti D, Weiner GM, Ares W, et al. Cerebral venous sinus thrombosis in pregnancy and puerperium: A pooled, systematic review. J Clin Neurosci. 2017;39:9-15.

2. Bousser MG, Ferro JM. Cerebral venous thrombosis: an update. Lancet Neurol. 2007;6(2):162-170.

3. Atanassova P, Massaldjieva R. Cerebral venous sinus thrombosis-diagnostic strategies and prognostic models: a review. Venous Thromb [Internet]. 2012 [cited 2017 Aug 21]; Available from: https:/www.intechopen.com/ download/pdf $/ 25536$

4. McCaulley JA, Pates JA. Postpartum cerebral venous thrombosis. Obstet Gynecol. 2011;118(2 Pt 2):423-425.

5. Femi OL, Mohammed H, Musa B, Ibrahim D, Gwaram B, Dutse A. Puerperal superior sagittal sinus thrombosis and pulmonary embolism: a case report and review of literature. J Basic Clin Reprod Sci [Internet]. [cited 2017 Aug 21];3(1). Available from: http://www.jbcrs. org/articles/puerperal-superior-sagittal-sinus-thrombosisand-pulmonary-embolism-a-case-report-and-review-ofliterature.pdf

6. Zachary H, Nathan L, Francis C. Cerebral venous sinus thrombosis. JSM Intern Med [Internet]. 2017;2(1). Available from: https:/www.jscimedcentral.com/InternalMedicine/internalmedicine-2-1003.pdf

7. Hundozi Z, Ibishi N, Jashari F, Rrustemi J, Gjikolli B. Superior sagittal sinus thrombosis presenting with hallucinations in the puerperium: a case report. Int J Biomed [Internet]. 2110 [cited 2017 Aug 21];10(64):294-297. Available from: http://www.ijbm.org/articles/IJBM_6(4)_CR2.pdf

8. Yamamoto J, Kakeda S, Takahashi M, Idei M, Nakano Y, Soejima Y, Saito T, et al. Severe subarachnoid hemorrhage associated with cerebral venous thrombosis in early pregnancy: a case report. J Emerg Med. 2013;45(6):849-855.

9. Panda S, Prashantha DK, Shankar SR, Nagaraja D. Localized convexity subarachnoid haemorrhage - a sign of early cerebral venous sinus thrombosis. Eur J Neurol. 2010;17(10):1249-1258.

10. Ko YP, Hsu CY, Yang CL, Cheng SJ, Tsai HJ, Chen CP, Hsu YW. Postcesarean subarachnoid hemorrhage as the initial presentation of cerebral venous thrombosis. Taiwan J Obstet Gynecol. 2007;46(3):317-319.

11. Cilingir IU, Ormeci T, Kaya B, Msrlolu M. A case of postpartum cerebral venous thrombosis presented by convulsions. [cited 2017 Aug 21]; Available from: http://www. perinataljournal.com/Files/Archive/en-US/Articles/PJ5fca04d5-a4b8-4540-a889-2855b40bf7c6.pdf

12. Damak M, Crassard I, Wolff V, Bousser MG. Isolated lateral sinus thrombosis: a series of 62 patients. Stroke. 2009;40(2):476-481.

13. van der Stege JG, Engelen MJ, van Eyck J. Uncomplicated pregnancy and puerperium after puerperal cerebral venous thrombosis. Eur J Obstet Gynecol Reprod Biol. 1997;71(1):99-100. 
14. Einhaupl KM, Villringer A, Meister W, Mehraein S, Garner C, Pellkofer M, Haberl RL, et al. Heparin treatment in sinus venous thrombosis. Lancet. 1991;338(8767):597600 .
15. Chisholm ME, Campbell DC. Postpartum postural headache due to superior sagittal sinus thrombosis mistaken for spontaneous intracranial hypotension. Can J Anaesth. 2001;48(3):302-304. 